\title{
Optical absorbtion by atomically doped carbon nanotubes
}

\author{
I.V. Bondarev and B. Vlahovic \\ Physics Department, North Carolina Central University, \\ 1801 Fayetteville Str, Durham, NC 27707, USA
}

\begin{abstract}
We analyze optical absorption by atomically doped carbon nanotubes with a special focus on the frequency range close to the atomic transition frequency. We derive the optical absorbtion line-shape function and, having analyzed particular achiral nanotubes of different diameters, predict the effect of absorbtion line splitting due to strong atom-vacuum-field coupling in small-diameter nanotubes. We expect this effect to stimulate relevant experimental efforts and thus to open a path to new device applications of atomically doped carbon nanotubes in modern nanotechnologies.
\end{abstract}

PACS numbers: 78.40.Ri, 73.22.-f, 73.63.Fg, 78.67.Ch

Carbon nanotubes $(\mathrm{CNs})$ are graphene sheets rolled-up into cylinders of approximately $1 \mathrm{~nm}$ in diameter. Extensive work carried out worldwide in recent years has revealed intriguing physical properties of these novel molecular scale wires [1, 2]. Nanotubes have been shown to be useful for miniaturized electronic, mechanical, electromechanical, chemical and scanning probe devices and as materials for macroscopic composites [3, 4]. Important is that their intrinsic properties may be substantially modified in a controllable way by doping with extrinsic impurity atoms, molecules and compounds [5, 6, 7, 8, 9]. Recent successful experiments on encapsulation of single atoms into single-wall $\mathrm{CNs}$ 8] and their intercalation into single-wall CN bundles [5, 9 ], along with numerous studies of monoatomic gas absorbtion by the CN bundles (see [10] for a review), stimulate an in-depth theoretical analysis of near-field electrodynamical properties of atomically doped CNs systems. This is of both fundamental and applied interest as it sheds light on the peculiarities of the atom-electromagnetic-field interactions in quasi-1D dispersive and absorbing surroundings, and, eventually, would open routes for new challenging nanophotonics applications of atomically doped CN systems as various sources of coherent light emitted by dopant atoms.

Typically, there may be two qualitatively different regimes of the interaction of an atomic state with a vacuum electromagnetic field in the vicinity of a CN 11, 12, 13, 14]. They are the weak coupling regime and the strong coupling regime. The former is characterized by the monotonic exponential decay dynamics of the upper atomic state with the decay rate altered compared with the free-space value. The latter is, in contrast, strictly non-exponential and is characterized by reversible Rabi oscillations where the energy of the initially excited atom is periodically exchanged between the atom and the field. We have recently shown that the relative density of photonic states (DOS) near the CN effectively increases due to the presence of additional surface photonic states coupled with $\mathrm{CN}$ electronic quasiparticle excitations [11. This causes the atom-vacuumfield coupling constant, which is determined by the lo- cal (distance-dependent) photonic DOS, to be very sensitive to the atom-CN-surface distance. At small enough distances, the system may exhibit strong atom-field coupling 12, 13], giving rise to rearrangement ("dressing") of atomic levels by the vacuum-field interaction with formation of atomic quasi-1D cavity polaritons. The problem of stability of quasi-1D polaritonic states in atomically doped CNs has been partly addressed in Refs. 13, 14] by investigating the atom-nanotube van der Waals interactions and demonstrating the inapplicability of standard weak-coupling-based van der Waals coupling models close to the CN surface. In this Letter, we analyze optical absorption properties of atomically doped $\mathrm{CNs}$ with a special focus on the frequency range close to the atomic transition frequency and predict the effect of the absorbtion line splitting for quasi-1D atomic polaritons in small-diameter CNs.

We consider a two-level atom positioned at the point $\mathbf{r}_{A}$ near an infinitely long achiral single-wall CN. The atom interacts with a quantum electromagnetic field via an electric dipole transition of frequency $\omega_{A}$. The atomic dipole moment, $\mathbf{d}=d_{z} \mathbf{e}_{z}$, is assumed to be directed along the $\mathrm{CN}$ axis (assigned by the unit vector $\mathbf{e}_{z}$ ) which is chosen to be the $z$-quantization axis of the system. The contribution of the transverse dipole moment orientation is suppressed because of the strong depolarization of the transverse field in an isolated CN (the so-called dipole antenna effect [15]). The total secondly quantized Hamiltonian of the system is given by 13,14

$$
\begin{aligned}
\hat{H} & =\int_{0}^{\infty} d \omega \hbar \omega \int d \mathbf{R} \hat{f}^{\dagger}(\mathbf{R}, \omega) \hat{f}(\mathbf{R}, \omega)+\frac{\hbar \tilde{\omega}_{A}}{2} \hat{\sigma}_{z} \\
& +\int_{0}^{\infty} d \omega \int d \mathbf{R}\left[\mathrm{g}^{(+)}\left(\mathbf{r}_{A}, \mathbf{R}, \omega\right) \hat{\sigma}^{\dagger}\right. \\
& \left.-\mathrm{g}^{(-)}\left(\mathbf{r}_{A}, \mathbf{R}, \omega\right) \hat{\sigma}\right] \hat{f}(\mathbf{R}, \omega)+\text { h.c. },
\end{aligned}
$$

with the three items representing the electromagnetic field (modified by the presence of the $\mathrm{CN}$ ), the two-level atom and their interaction, respectively. The operators $\hat{f}^{\dagger}(\mathbf{R}, \omega)$ and $\hat{f}(\mathbf{R}, \omega)$ are the scalar bosonic field operators defined on the surface of the $\mathrm{CN}$ ( $\mathbf{R}$ is the radiusvector of an arbitrary point of the $\mathrm{CN}$ surface). They play 
the role of the fundamental dynamical variables of the field subsystem and satisfy the standard bosonic commutation relations. The Pauli operators, $\hat{\sigma}_{z}=|u\rangle\langle u|-| l\rangle\langle l|$, $\hat{\sigma}=|l\rangle\langle u|$ and $\hat{\sigma}^{\dagger}=|u\rangle\langle l|$, describe the atomic subsystem and electric dipole transitions between the two atomic states, upper $|u\rangle$ and lower $|l\rangle$, separated by the transition frequency $\omega_{A}$. This (bare) frequency is modified by the diamagnetic $\left(\sim \mathbf{A}^{2}\right)$ atom-field interaction yielding the new renormalized transition frequency $\tilde{\omega}_{A}=\omega_{A}\left[1-2 /\left(\hbar \omega_{A}\right)^{2} \int_{0}^{\infty} d \omega \int d \mathbf{R}\left|\mathrm{g}^{\perp}\left(\mathbf{r}_{A}, \mathbf{R}, \omega\right)\right|^{2}\right]$ in the second term of the Hamiltonian. The dipole atom-field interaction matrix elements $\mathrm{g}^{( \pm)}\left(\mathbf{r}_{A}, \mathbf{R}, \omega\right)$ are given by $\mathrm{g}^{( \pm)}=\mathrm{g}^{\perp} \pm\left(\omega / \omega_{A}\right) \mathrm{g}^{\|}$where $\mathrm{g}^{\perp(\|)}\left(\mathbf{r}_{A}, \mathbf{R}, \omega\right)=-i\left(4 \omega_{A} / c^{2}\right)$ $\sqrt{\pi \hbar \omega \operatorname{Re} \sigma_{z z}(\omega)} d_{z}{ }^{\perp(\|)} G_{z z}\left(\mathbf{r}_{A}, \mathbf{R}, \omega\right)$ with ${ }^{\perp(\|)} G_{z z}$ being the $z z$-component of the transverse (longitudinal) Green tensor (with respect to the first variable) of the electromagnetic subsystem and $\sigma_{z z}(\omega)$ representing the CN surface axial conductivity. The matrix elements $\mathrm{g}^{\perp(\|)}$ have the property of $\int d \mathbf{R}\left|\mathrm{g}^{\perp(\|)}\left(\mathbf{r}_{A}, \mathbf{R}, \omega\right)\right|^{2}=$ $\left(\hbar^{2} / 2 \pi\right)\left(\omega_{A} / \omega\right)^{2} \Gamma_{0}(\omega) \xi^{\perp(\|)}\left(\mathbf{r}_{A}, \omega\right)$ with $\xi^{\perp(\|)}\left(\mathbf{r}_{A}, \omega\right)=$ $\operatorname{Im}^{\perp(\|)} G_{z z}^{\perp(\|)}\left(\mathbf{r}_{A}, \mathbf{r}_{A}, \omega\right) / \operatorname{Im} G_{z z}^{0}(\omega)$ being the transverse (longitudinal) distance-dependent (local) photonic DOS functions and $\Gamma_{0}(\omega)=8 \pi \omega^{2} d_{z}^{2} \operatorname{Im} G_{z z}^{0}(\omega) / 3 \hbar c^{2}$ representing the atomic spontaneous decay rate in vacuum where $\operatorname{Im} G_{z z}^{0}(\omega)=\omega / 6 \pi c$ is the vacuum imaginary Green tensor $z z$-component. The Hamiltonian (11) involves only two standard approximations: the electric dipole approximation and two-level approximation. The rotating wave approximation commonly used is not applied, and the diamagnetic term of the atom-field interaction is not neglected (as opposed to, e.g., Refs. 11, 12, 16]). Quantum electrodynamics (QED) of the two-level atom close to the $\mathrm{CN}$ is thus described in terms of only two intrinsic characteristics of the electromagnetic subsystem - the transverse and longitudinal local photonic DOS functions.

In deriving the absorbtion/emission spectral line shape, we follow the general quantum approach of Ref. 17]. (Obviously, the absorbtion line shape coincides with the emission line shape if the monochromatic incident light beam is being used in the absorbtion experiment.) When the atom is initially in the upper state and the field subsystem is in vacuum, the time-dependent wave function of the whole system can be written as

$$
\begin{aligned}
|\psi(t)\rangle & =C_{u}(t) e^{-i\left(\tilde{\omega}_{A} / 2\right) t}|u\rangle|\{0\}\rangle \\
& +\int d \mathbf{R} \int_{0}^{\infty} d \omega C_{l}(\mathbf{R}, \omega, t) e^{-i\left(\omega-\tilde{\omega}_{A} / 2\right) t}|l\rangle|1(\mathbf{R}, \omega)\rangle,
\end{aligned}
$$

where $|\{0\}\rangle$ is the vacuum state of the field subsystem, $|\{1(\mathbf{R}, \omega)\}\rangle$ is its excited state with the field being in the single-quantum Fock state, and $C_{u}$ and $C_{l}$ are the population probability amplitudes of the upper state and the lower state of the whole system, respectively. Applying the Hamiltonian (1) to the wave function (2), one has

$$
\dot{C}_{u}(t)=-\frac{i}{\hbar} \int_{0}^{\infty} d \omega \int d \mathbf{R} \mathrm{g}^{(+)}\left(\mathbf{r}_{A}, \mathbf{R}, \omega\right)
$$

$$
\begin{gathered}
\times C_{l}(\mathbf{R}, \omega, t) e^{-i\left(\omega-\tilde{\omega}_{A}\right) t}, \\
\dot{C}_{l}(\mathbf{R}, \omega, t)=-\frac{i}{\hbar}\left[\mathrm{g}^{(+)}\left(\mathbf{r}_{A}, \mathbf{R}, \omega\right)\right]^{*} C_{u}(t) e^{i\left(\omega-\tilde{\omega}_{A}\right) t} .
\end{gathered}
$$

In terms of the probability amplitudes above, the emission/absorbtion spectral line shape $I(\omega)$ is given by $\int d \mathbf{R}\left|C_{l}(\mathbf{R}, \omega, t \rightarrow \infty)\right|^{2}$, yielding, after the substitution of $C_{l}$ obtained by integration [under initial conditions $\left.C_{l}(\mathbf{R}, \omega, 0)=0, C_{u}(0)=1\right]$ of Eq. (44), the result

$$
\tilde{I}(x)=\tilde{I}_{0}(x)\left|\int_{0}^{\infty} d \tau C_{u}(\tau) e^{i\left(x-\tilde{x}_{A}\right) \tau}\right|^{2},
$$

where $\tilde{I}_{0}(x)=\left[\tilde{\Gamma}_{0}(x) / 2 \pi\right]\left[\left(x_{A} / x\right)^{2} \xi^{\perp}(x)+\xi^{\|}(x)\right]$ and we have, for convenience, introduced the dimensionless variables $\tilde{I}=2 \gamma_{0} I / \hbar, \tilde{\Gamma}_{0}=\hbar \Gamma_{0} / 2 \gamma_{0}, x=\hbar \omega / 2 \gamma_{0}$, and $\tau=2 \gamma_{0} t / \hbar$ with $\gamma_{0}=2.7 \mathrm{eV}$ being the carbon nearest neighbor hopping integral appearing in the $\mathrm{CN}$ surface axial conductivity $\sigma_{z z}$.

The upper state population probability amplitude $C_{u}(\tau)$ in Eq. (5) is given by the Volterra integral equation [obtained by substituting the result of the formal integration of Eq. (4) into Eq. (3)] with the kernel determined by the local photonic DOS functions $\xi^{\perp(\|)}\left(\mathbf{r}_{A}, x\right)[12,13]$. Thus, the numerical solution is only possible for the line shape $\tilde{I}(x)$, strictly speaking. This, however, offers very little physical insight into the problem of optical absorbtion by atomically doped CNs under different atom-field coupling regimes. Therefore, we choose a simple analytical approach valid for those atomic transition frequencies $\tilde{x}_{A}$ which are located in the vicinity of the resonance frequencies $x_{r}$ of the DOS functions $\xi^{\perp(\|)}$. Within this approach, $\xi^{\perp(\|)}\left(\mathbf{r}_{A}, x \sim x_{r}\right)$ are approximated by the Lorentzians of the same half-width-at-half-maxima $\delta x_{r}$, thus making it possible to solve the integral equation for $C_{u}$ analytically to obtain 12, 13 .

$$
\begin{aligned}
C_{u}(\tau) & \approx \frac{1}{2}\left(1+\frac{\delta x_{r}}{\sqrt{\delta x_{r}^{2}-X^{2}}}\right) e^{-\frac{1}{2}\left(\delta x_{r}-\sqrt{\delta x_{r}^{2}-X^{2}}\right) \tau} \\
& +\frac{1}{2}\left(1-\frac{\delta x_{r}}{\sqrt{\delta x_{r}^{2}-X^{2}}}\right) e^{-\frac{1}{2}\left(\delta x_{r}+\sqrt{\delta x_{r}^{2}-X^{2}}\right) \tau}
\end{aligned}
$$

with

$$
X=\sqrt{2 \delta x_{r} \tilde{\Gamma}_{0}\left(\tilde{x}_{A}\right) \xi^{\perp}\left(\mathbf{r}_{A}, \tilde{x}_{A}\right)} .
$$

This solution is valid for $\tilde{x}_{A} \approx x_{r}$ whatever the atom-field coupling strength is, yielding the exponential decay of the upper state population, $\left|C_{u}(\tau)\right|^{2} \approx \exp \left[-\tilde{\Gamma}\left(\tilde{x}_{A}\right) \tau\right]$ with the rate $\tilde{\Gamma} \approx \tilde{\Gamma}_{0} \xi^{\perp}$, in the weak coupling regime where $\left(X / \delta x_{r}\right)^{2} \ll 1$, and the decay via damped Rabi oscillations, $\left|C_{u}(\tau)\right|^{2} \approx \exp \left(-\delta x_{r} \tau\right) \cos ^{2}(X \tau / 2)$, in the strong coupling regime where $\left(X / \delta x_{r}\right)^{2} \gg 1$.

Substituting Eq. (6) into Eq. (5) and integrating over $\tau$, one arrives at the expression

$$
\tilde{I}(x)=\tilde{I}_{0}\left(\tilde{x}_{A}\right) \frac{\left(x-\tilde{x}_{A}\right)^{2}+\delta x_{r}^{2}}{\left[\left(x-\tilde{x}_{A}\right)^{2}-X^{2} / 4\right]^{2}+\delta x_{r}^{2}\left(x-\tilde{x}_{A}\right)^{2}}
$$


representing the emission/absorbtion spectral line shape for frequencies $x \sim x_{r} \approx \tilde{x}_{A}$ regardless of the atom-field coupling strength. The line shape is clearly seen to be of a symmetric two-peak structure in the strong coupling regime where $\left(X / \delta x_{r}\right)^{2} \gg 1$. The exact peak positions are $x_{1,2}=\tilde{x}_{A} \pm(X / 2) \sqrt{\sqrt{1+8\left(\delta x_{r} / X\right)^{2}}-4\left(\delta x_{r} / X\right)^{2}}$, separated from each other by $x_{1}-x_{2} \sim X$ with the Rabi frequency $X$ given by Eq. (7). In the weak coupling regime $\left(X / \delta x_{r}\right)^{2} \ll 1$, and $x_{1,2}$ become complex, indicating that there are no longer peaks at these frequencies. As this takes place, Eq. (8) is approximated with the weak coupling condition, the fact that $x \sim \tilde{x}_{A}$ and Eq. (7), to give the well-known Lorentzian $\tilde{I}(x)=$ $\tilde{I}_{0}\left(\tilde{x}_{A}\right) /\left[\left(x-\tilde{x}_{A}\right)^{2}+\tilde{\Gamma}^{2}\left(\tilde{x}_{A}\right) / 4\right]$ of the half-width-at-halfmaximum $\tilde{\Gamma}\left(\tilde{x}_{A}\right) / 2$, peaked at $x=\tilde{x}_{A}$.

To compute the absorbtion line shapes of particular $\mathrm{CNs}$, one needs to know their transverse local photonic DOS functions $\xi^{\perp}\left(\mathbf{r}_{A}, \tilde{x}_{A}\right)$ in Eqs. (7) and (8). These are basically determined by the CN surface axial conductivity $\sigma_{z z}$. The latter one was calculated in the relaxationtime approximation (relaxation time $3 \times 10^{-12} \mathrm{~s}$ ) at temperature $300 \mathrm{~K}$. The function $\xi^{\perp}$ was calculated thereafter as described in Refs. 11, 12, 13, 14]. The vacuum spontaneous decay rate was estimated from the expression $\tilde{\Gamma}_{0}(x)=\alpha^{3} x(\alpha=1 / 137$ is the fine-structure constant) valid for atomic systems with Coulomb interaction [18].

Figure 1shows the computed $\xi^{\perp}(x)$ and normalized optical absorption spectra $A(x)=\tilde{I}(x) / \tilde{I}_{\text {peak }}$ for the atom close to the $(9,0) \mathrm{CN}$. When the atom, being outside, approaches the $\mathrm{CN}$ surface, the function $\xi^{\perp}$ goes up [shown in Fig. 1(a); the vertical dashed lines indicate the DOS peak frequencies for which $A(x)$ in Figs. 1(b) and प(c) are calculated] due to the increasing role of additional surface photonic modes of the nanotube. This causes the atomvacuum-field coupling to increase with the effect [shown in Fig. 1(b)] of first broadening and then, when the strong atom-field coupling regime is realized, splitting the initial Lorentzian line into two symmetric components separated by the Rabi frequency $X \approx 0.1$ corresponding to the energy $0.1 \times 2 \gamma_{0}=0.54 \mathrm{eV}$. In Fig. I(c) much stronger Rabi splitting of $X \approx 0.3$ is demonstrated (Rabi energy $0.3 \times 2 \gamma_{0}=1.62 \mathrm{eV}$ ) for the atom located in the center of the CN. This is in agreement with the fact that, due to the nanotube curvature, the effective interaction area between the atom and the $\mathrm{CN}$ surface is larger when the atom is inside rather when it is outside the CN [13, 14].

Figure 2(a) presents $\xi^{\perp}(x)$ for the atom in the center of the $(5,5),(10,10)$ and $(23,0)$ CNs. It is seen to decrease with increasing the $\mathrm{CN}$ radius, representing the decrease of the atom-field coupling strength as the atom moves away from the $\mathrm{CN}$ wall. To calculate the normalized absorbtion curves $A(x)=\tilde{I}(x) / \tilde{I}_{\text {peak }}$ in this case, we have fixed $\tilde{x}_{A}=0.45$ [indicated by the vertical dashed line in Fig. 2(a)] because this is the approximate peak posi-
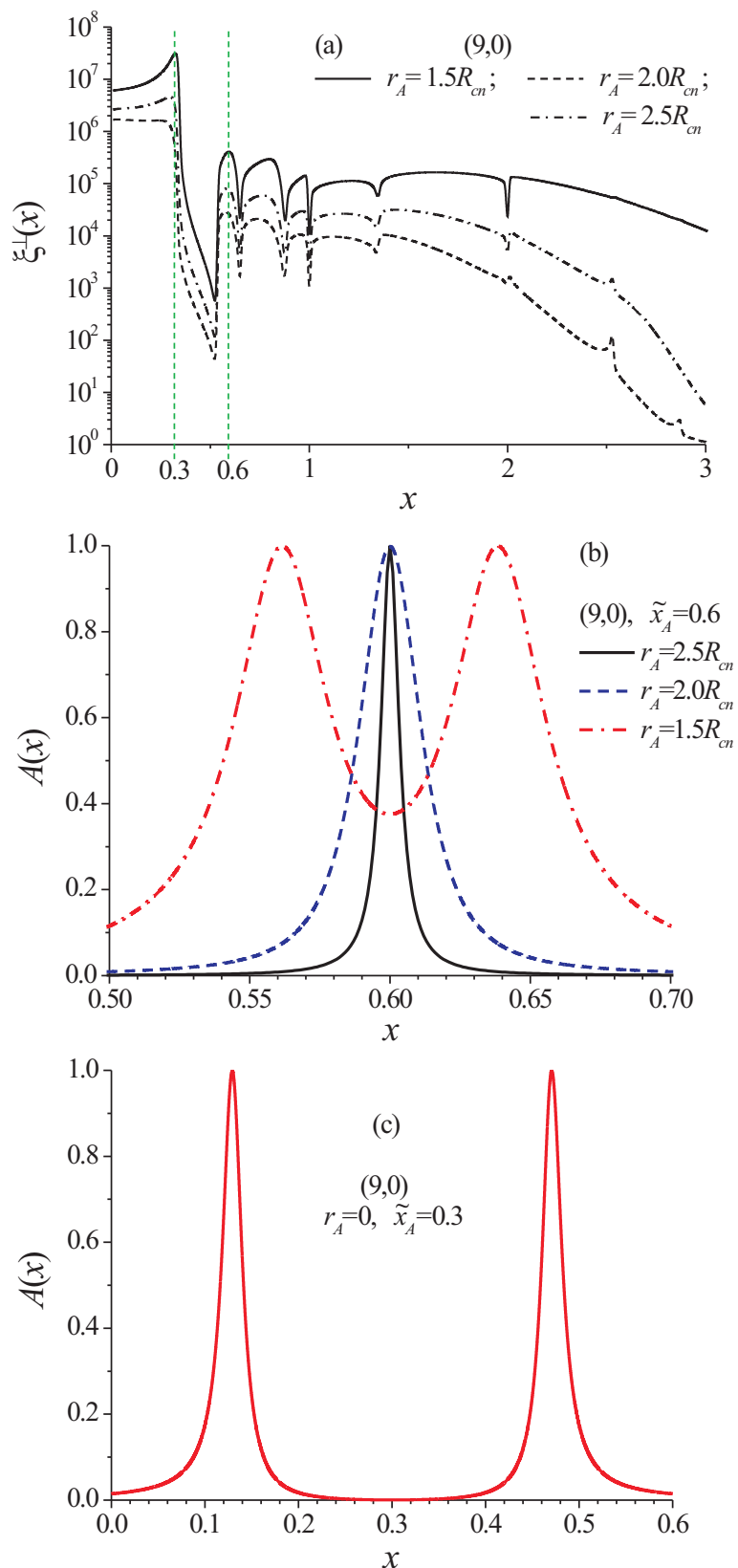

FIG. 1: (Color online) Transverse local photonic DOS (a) and normalized absorbtion line shapes (b) for the atom at different distances outside the $(9,0) \mathrm{CN}$. (c) Normalized absorbtion line shape for the atom in the center of the $(9,0) \mathrm{CN}$.

tion of $\xi^{\perp}$ for all the three CNs. The result is shown in Fig. 2(b). For the small radius $(5,5) \mathrm{CN}$, the absorbtion line is split into two components, indicating the strong atom-field coupling with the Rabi splitting $X \approx 0.2$ corresponding to the energy of $0.2 \times 2 \gamma_{0}=1.08 \mathrm{eV}$. The splitting is disappearing with increasing $\mathrm{CN}$ radius, and the absorbtion line shape becomes Lorentzian with the narrower widths for the larger radii CNs.

Recently, Rabi splitting $\sim 140-400 \mu \mathrm{eV}$ was detected 

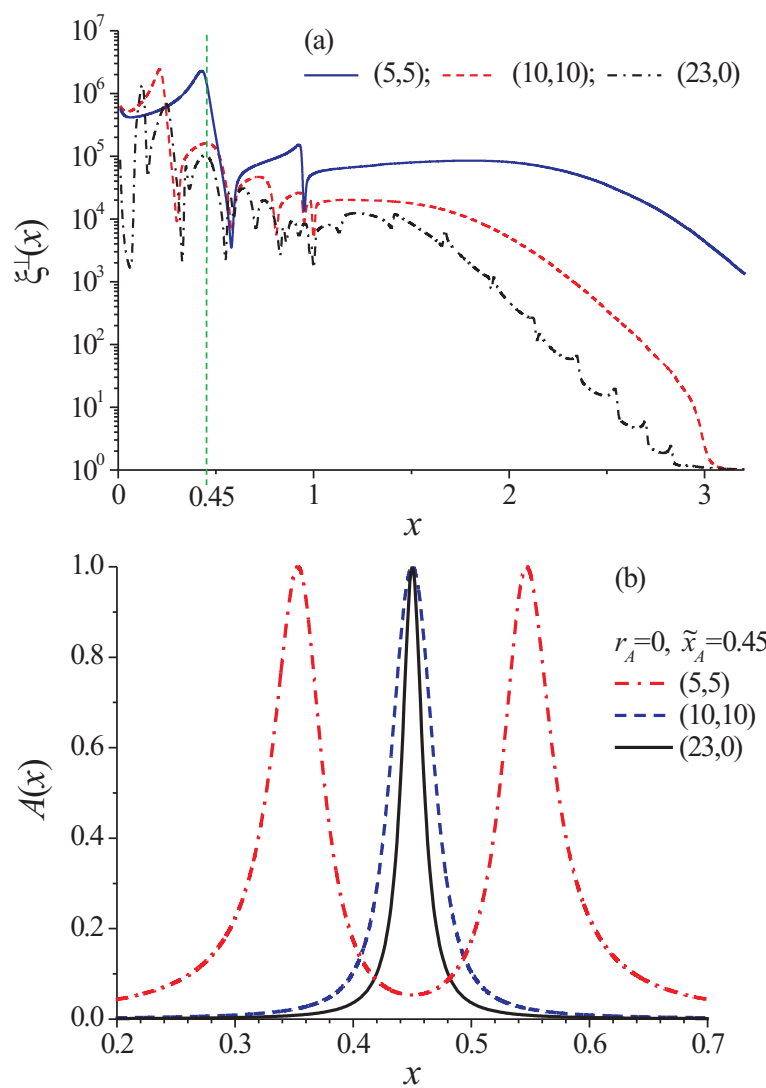

FIG. 2: (Color online) Transverse local photonic DOS (a) and normalized absorbtion line shapes (b) for the atom in the center of the $\mathrm{CNs}$ of increasing radii.

in the photoluminescence experiments for quasi-0D excitonic polaritons in quantum dots in semiconductor microcavities 19, 20, 21]. The effect we report in this Letter is at least 3 orders of magnitude larger. This comes from the fact that the typical atomic binding energies, are at least 3 orders of magnitude larger than the typical excitonic binding energies in solids. In terms of the cavity-QED (see, e.g., Ref. 22]), the coupling constant of the atom-cavity interaction is given in our notations by $\hbar g=d_{z}\left(2 \pi \hbar \tilde{\omega}_{A} / \tilde{V}\right)^{1 / 2}$ with $\tilde{V}$ being the effective volume of the field mode the atom interacts with. In our case $\tilde{V} \sim \pi R_{c n}^{2}\left(\tilde{\lambda}_{A} / 2\right)$, which is $\sim 10^{2} \mathrm{~nm}^{3}=10^{-7} \mu \mathrm{m}^{3}$ for the CNs with diameters $\sim 1 \mathrm{~nm}$ in the optical spectral region (as apposed to $\sim 10^{-1} \div 10^{-2} \mu \mathrm{m}^{3}$ for semiconductor microcavities in Refs. [19, 20, 21]). Small mode volumes yield large atom-cavity coupling constants in smalldiameter CNs. For example, in the situation shown in Fig. 2(a) one has $\hbar g \sim 0.3 \mathrm{eV}$ for the atom in the center of the $(5,5) \mathrm{CN}\left[R_{c n}=0.34 \mathrm{~nm}, d_{z} \sim e r \sim e\left(e^{2} / \hbar \tilde{\omega}_{A}\right)\right]$, whereas the "cavity" linewidth, which is related to the Purcell factor $\xi^{\perp}\left(\tilde{\omega}_{A}\right)$ via the mode quality factor when $\tilde{\omega}_{A} \approx \omega_{r}$ [22], is $\hbar \gamma_{c}=6 \pi \hbar c^{3} / \tilde{\omega}_{A}^{2} \xi^{\perp}\left(\tilde{\omega}_{A}\right) \tilde{V} \sim 0.03$, so that the strong coupling condition $g / \gamma_{c} \gg 1$ has been well satisfied.
To summarize, we have shown that, similar to semiconductor microcavities and photonic band-gap materials, carbon nanotubes may qualitatively change the character of the atom-electromagnetic-field interaction, yielding strong atom-field coupling. We predict the effect of the optical absorbtion line splitting of $\sim 1 \mathrm{eV}$ in the vicinity of the energy of the atomic transition for quasi-1D atomic polaritons in small-diameter atomically doped CNs. We expect this effect to stimulate relevant experimental efforts and thereby to open a path to novel device applications of atomically doped CNs. For example, quasi-0D excitonic polaritons in quantum dots in semiconductor microcavities were suggested recently to be a possible way to produce the excitonic qubit entanglement [23]. Leaving the details for a forthcoming publication, we conclude that, being strongly coupled to the (resonator-like) cylindrical nanotube environment, the two atomic quasi1D polaritons can be entangled as well, thus challenging novel applications of atomically doped CNs in quantum communication technologies.

This work was supported by DoD and NASA via grants No W911NF-05-1-0502 and NAG3-804.

* Also at: Institute for Nuclear Problems, Belarusian State University, Bobruiskaya Str.11, 220050 Minsk, Belarus

[1] R.Saito, G.Dresselhaus, and M.S.Dresselhaus, Science of Fullerens and Carbon Nanotubes (Imperial College Press, London, 1998).

[2] H.Dai, Surf. Sci. 500, 218 (2002).

[3] R.H.Baughman, A.A.Zakhidov, and W.A.de Heer, Science 297, 787 (2002).

[4] S.G.Chou et al., Phys. Rev. Lett. 94, 127402 (2005).

[5] L.Duclaux, Carbon 40, 1751 (2002).

[6] S.Latil, F.Triozon, and S.Roche, Phys. Rev. Lett. 95, 126802 (2005).

[7] Y.-W.Son et al., Phys. Rev. Lett. 95, 216602 (2005).

[8] G.-H.Jeong et al., Phys. Rev. B 68, 075410 (2003).

[9] H.Shimoda et al., Phys. Rev. Lett. 88, 015502 (2002).

[10] M.M.Calbi et al., Rev. Mod. Phys. 73, 857 (2001).

[11] I.V.Bondarev et al., Phys. Rev. Lett. 89, 115504 (2002).

[12] I.V.Bondarev and Ph.Lambin, Phys. Rev. B 70, 035407 (2004); Phys. Lett. A 328, 235 (2004).

[13] I.V.Bondarev and Ph.Lambin, in: Trends in Nanotubes Reasearch (Nova Science, New York, 2006).

[14] I.V.Bondarev and Ph.Lambin, Phys. Rev. B 72, 035451 (2005); Solid State Commun. 132, 203 (2004).

[15] A.Jorio et al., Phys. Rev. B 65, 121402R(2002).

[16] H.T.Dung, L.Knöll, and D.-G.Welsch, Phys. Rev. A 62, 053804 (2000).

[17] W.Heitler, The quantum Theory of Radiation (Clarendon, Oxford, 1954).

[18] A.S.Davydov, Quantum Mechanics (NEO, Ann Arbor, MI, 1967).

[19] J.P.Reithmaier et al., Nature 432, 197 (2004).

[20] T.Yoshie et al., Nature 432, 200 (2004).

[21] E.Peter et al., Phys. Rev. Lett. 95, 067401 (2005).

[22] L.C.Andreani, G.Panzarini, and J.-M.Gérard, Phys. Rev. 
B 60, 13276 (1999).

[23] S.Hughes, Phys. Rev. Lett. 94, 227402 (2005). 